\title{
A Survey on Load Balanced Energy Efficient Clustering Protocols for Wireless Sensor Networks
}

\author{
Manpreet Kaur \\ Department of Computer Science and Engineering \\ Baba Banda Singh Bahadur Engineering College, \\ Fatehgarh Sahib, Punjab, India
}

\author{
Sandeep Kaur Dhanda \\ Department of Computer Science and Engineering \\ Baba Banda Singh Bahadur Engineering College, \\ Fatehgarh Sahib, Punjab, Indi
}

\begin{abstract}
A Large number of tiny sensors are equipped in a network termed as Wireless Sensor Network which can be an effective tool for gathering of data from environment. Data which is collected through sensors are forwarded to the Base Station and from there to the end User. In WSN, energy efficiency is the primary factor that requires consideration. Considering this fact, clustering is introduced in WSN as it provides effective data aggregation as well as scalability for large WSNs. Moreover, clustering is also helpful in conserving limited energy resources of the sensors involved in network. In addition to this, this paper reviewed several clustering algorithms exists in WSNs. Furthermore, several challenges faced in clustering are also highlighted in this paper.
\end{abstract}

\section{General Terms}

Different types Clustering Algorithm, Challenges of Clustering Algorithm

\section{Keywords}

Wireless Sensor Networks (WSNs), Clustering Algorithms, Energy Efficiency

\section{INTRODUCTION}

Sensor nodes or self-organized nodes are scattered in a region considered as wireless sensor network (WSN). In this network, huge number of nodes are involved that collects data from remote locations while interacting with physical event make this technology as most prominent. As the sensor network has dynamic property, so designing of an energy efficient routing protocol is the necessity. The whole network has subdivided into areas termed as cluster where a node has been is selected from number of other nodes and named as cluster head. This cluster head in the network communicates with the base station. Selection of cluster head is itself a research challenge which includes several factors likewise, Distance from Base station, Density of node and Battery consumption of a node etc. The significance of introducing the concept of clusters for transmitting data to the base station is to reduce total distance covered by individual nodes to the base station. In order to reduce energy consumption and increased scalability, Hierarchical clustering technique is used. Low Energy Adaptive Clustering Approach (LEACH) is a Hierarchical Clustering protocol specifically designed for proactive networks where communication takes place periodically. In hierarchical routing, two layer routing is drawn in. Cluster head Selection is performed in the first layer of routing and other is used for data transmission [9].

LEACH routing protocol breaks its operation in two rounds i.e. cluster head selection phase and routing phase. Once the node is elected as a cluster head for present round, then their status has been advertised to other nodes that took part in sensing task. After receiving information, rest of the nodes decides to which cluster they want to belong. The cluster head distribution should be uniform which formulates this network as a desirable technique. When a particular cluster in the network has completed its formation then it further creates TDMA schedule for its own member nodes. This schedule explains which member node sends its sensed data to the dedicated cluster head and for rest of the period; it continues its sleep mode. The upside of TDMA based MAC protocol is that it provides collision free approach and utilization of channel completely through individual member. Cluster head gathered all the data from non-cluster head nodes process it and then transmit this compressed data to the base station. In existing LEACH, TDMA scheme is used for scheduling where the bandwidth is pre-located. Several variants of LEACH have been introduced for better energy efficiency[14]. Some of them are discussed in this paper. The actual sensor nodes are arranged directly into different groupings in clustered based WSN, termed as clusters shown in figure 1.

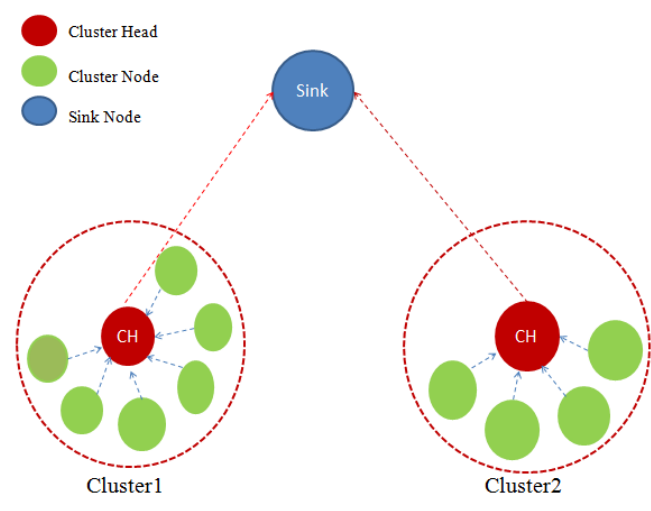

Fig1: Model of Wireless Sensor Networks

\section{CLUSTERING ALGORITHMS}

This section of the paper classified and analyzed several effective clustering algorithms for WSNs.

\subsection{Low-Energy Adaptive Clustering Hierarchy (LEACH)}

This algorithm uses a distributed algorithm to form a cluster and other nodes take autonomous decisions without considering a centralized control. The process goes with the introduction of cluster head in the network with a probability $\mathrm{p}$ and broadcasts its decision to other nodes. Each non- $\mathrm{CH}$ node chooses the $\mathrm{CH}$ in terms of determining its cluster so that least communication energy can be reached. In each round, the $\mathrm{CH}$ has been changed and to balance the energy usage in the network random rotation of $\mathrm{CHs}$ are performed. The clusters in this network are formed on the basis of received signal strength and $\mathrm{CH}$ nodes are treated as routers to the base station. All data processing like Data fusion and 
aggregation are local to the cluster [16]. The key areas of LEACH protocol are as:

1. The overhead of making decision regarding which node becomes cluster head is wasted as individual node in the network decides independent of other nodes.

2. Clusters are operated independently due to CDMA, as each cluster in the network assigned with different code.

3. The minimum transmission energy is calculated through individual node for communication with its cluster head and this power level is considered only for transmission.

In $\mathrm{LEACH}$, changing of $\mathrm{CH}$ is probabilistic and a node with low energy gets selected as a $\mathrm{CH}$ easily. The whole cluster becomes non functional when this node dies out. LEACH technique forms one-hop intra and inter cluster topology in which member node can transmit the data directly to the $\mathrm{CH}$. This aggregated data is then forwarded to the base station. One of the downside of this protocol is that it is not applicable to networks employed in large regions. If $\mathrm{CHs}$ are far from the base station, in that case, LEACH will not be applicable. In order to overcome the issues of this algorithm, several techniques have been proposed such as PEGASIS, EEPSC, TEEN, MECH etc.

\subsection{Two-Level LEACH (TL-LEACH)}

This algorithm is a proposed extension of LEACH algorithm. Basically, two levels of cluster heads are involved in this technique referred as primary and secondary. The primary cluster head in individual cluster communicates with the secondary and then this secondary node will communicate with other nodes in the cluster. Additionally, data fusion process is similar to that performed in LEACH and communication within a cluster is scheduled using TDMA as in LEACH. The process is followed through the selection of primary and secondary cluster heads similar to $\mathrm{LACH}$ protocol. The selection of primary and secondary cluster head is through the prior probability then communication is taken place. From source node to sink node, communication has performed using following steps:

1. Initially, secondary nodes collect data from other nodes in a cluster and performed data fusion.

2. Secondly, primary nodes aggregates data from secondary clusters and performed data fusion.

The introduction of TL-LEACH protocol has the ability of reducing number of nodes for transmission to the base station and total utilization of energy. This protocol is also not applicable where $\mathrm{CH}$ is far from the base station[15].

\subsection{Energy Efficient Clustering Scheme (EECS)}

This protocol is considered as a clustering algorithm where

cluster heads compete to enhance the ability of energy for a particular round. In this technique, candidates broadcast their remaining energy to other neighboring candidates. The node will become a cluster head if any of the given nodes does not find a node with more residual energy. The formation of clusters in this protocol is different from other clustering algorithms such as LEACH [10]. In this algorithm, dynamic sizing of clusters is based upon the cluster distance from the base station. However, this will lead to a problem of high consumption of energy as greater range will require more energy for transmission in comparison with those that are closer. Consequently, distribution of energy among different nodes will extend lifetime of the network and improved resource usage. On the counterpart, clusters those are closer to the base station will become congested and may resultant into earlier $\mathrm{CH}$ death.

\subsection{Energy-efficient unequal clustering (EEUC)}

The problem of hot spot exists in multi-hop WSNs in which $\mathrm{CHs}$ those are closer to the base station die faster as they relay more traffic in comparison with other remote nodes. Considering this fact, a novel approach named as Energy Efficient Unequal Clustering protocol has proposed in view of balancing the consumption of energy. In this protocol, consumption of energy has reduced in inter-cluster and intracluster as size of the cluster near the sink is smaller than the clusters which are far from the sink node. EEUC algorithm is similar to EECS technique as it is also distance based scheme. Moreover, individual node in this network has global knowledge i.e. its locations and distances to the sink node. This technique provides improved network lifetime and balance the overall load among nodes. In addition to this, this protocol has the capability of resolving hot spot problems as the size of cluster is proportional to the distance to base station. However, it has also been suffering from several issues such as global data aggregation of all sensors becomes overhead for the network which degrades performance of the network specifically for a multi-hop network [4].

\subsection{Multihop routing protocol with unequal clustering (MRPUC)}

It is a distributed clustering scheme performed in rounds and individual round has been divided into three phases such as cluster setup, inter-cluster Multihop routing information and last round is data transmission. Each node in the network collects correlative information of its neighbour nodes and a node with maximum residual energy is considered as a cluster head. Cluster heads which are closer to the Base Station have small clusters for heavy inter cluster forwarding task in view of saving energy for them. The regular nodes join particular clusters where there is more residual energy of cluster heads and are closer to them. An inter-cluster routing tree is taken as a network backbone where data is transmitted from $\mathrm{CHs}$ to the Base Station through multi-hop. As inter cluster communication depends upon residual energy which resultant into earlier death of Cluster Heads. Cluster heads route the data to the neighboring cluster head with highest residual energy[3]. The negative aspect of inter-cluster multi hop routing formation is that it may cause an additional overhead.

\subsection{Power-efficient and adaptive clustering hierarchy(PEACH)}

The primary issue which requires consideration in existing clustering protocols is high consumption of energy, cluster formation overhead and fixed level clustering. In view of resolving this problem, Power-Efficient and Adaptive Clustering Hierarchy protocol i.e. PEACH has been proposed for Wireless Sensor Networks. This protocol has an ability of minimizing the energy and maximizing the lifetime of the network. The process of cluster formation in PEACH is performed using overhearing characteristics of wireless communication in view of supporting adaptive multilevel clustering and avoids additional overheads. The overhearing a node can identify the source and neighbour nodes transmitted the packets at the destination. PEACH algorithm can be applicable in both location-unaware and location aware sensor 
networks[13]. The consumption of energy of each node has reduced on the basis of its overhearing characteristics and enhances the network lifetime.

\section{CHALLENGES FOR CLUSTERING ALGORITHMS}

Clustering schemes play a significant part in Wireless sensor network which can improve the performance of the network. There are several limitations in Wireless Sensor Networks which must be considering before propping clustering schemes.

\subsection{Limited Energy}

Sensor nodes in the wireless networks are smaller in size and battery operated. Thus they have limited amount of energy storage. After exhaustion of these batteries, it is not practicable to recharge or replace them. In comparison with the direct routing algorithms, clustering algorithms are more energy efficient. The consumption of energy is balanced in sensor nodes while optimizing the formation of cluster and reelect the Cluster heads on the basis of their residual energy, inter-cluster and efficient intra-cluster communication.

\subsection{Network Lifetime}

Limitation on energy of nodes resultant into limited network lifetime for nodes in a network. Clustering schemes in the network helps to enhance the lifetime while reducing the consumption of energy communication within and outside of clusters.

\subsection{Limited Abilities}

Small amount of stored energy limits several abilities of nodes such as processing, memory, storage and communication.

\subsection{Secure Communication}

The security of communication in wireless sensor network is very crucial while considering these networks for military applications. Consequently, establishment of secure as well as intra-cluster and inter cluster communication is considered as challenges in designing clustering algorithms as tiny nodes can be unattended while deploying.

\subsection{Cluster formation and $\mathrm{CH}$ selection}

These two parameters are considered as important operations in clustering algorithms. The application of clustering in WSN can reduce the wastage of energy and direct transmission between sensors and a base station can also be avoided. The scalability of WSN enhances through clustering in real world applications. In designing the clustering algorithms, several parameters such as optimum cluster size, cluster maintenance, election and re-election of cluster heads are required consideration. Thus, energy utilization can be maximized by selecting optimum criteria to isolate clusters and to choose the Cluster Heads.

\subsection{Synchronization}

In clustering scheme, synchronization as well as scheduling impacts overall performance of the network. TDMA in WSNs is used to schedule sleep intervals in order to reduce the consumption of energy. Therefore, such schemes required synchronization mechanisms which can set up as well as maintain the schedule of the transmission.

\subsection{Data Aggregation}

This parameter reduces duplication of data in the network. It can be possible in the large network that multiple nodes can sense similar information. Data Aggregation is used to differentiate sensed data and useful data. There are several clustering schemes that provide the capability of data aggregation and a suitable selection of clustering approach must be performed carefully.

\subsection{Repair Mechanisms}

As wireless sensor network is dynamic in nature, so it is prone to node mobility, interference, node death and delay. These situations can be resultant into link failure. Thus, while designing clustering schemes, the mechanisms need consideration to ensure link recovery and reliable data communication.

\subsection{Quality of Service (QoS)}

While considering overall network standpoint, QoS requirements in WSN can be considered briefly. Many of QoS requirements are application dependent (Acceptable delay and packet loss tolerance). Most of the existing clustering algorithms focused on energy efficient network utilization. Consequently, less attention has been given to QoS support in WSN due to which this parameter must be taken into account while designing.

\section{LITERATURE REVIEW}

Nayebi and Sarbazi-Azad proposed a novel scheme which was used to balance the consumption of energy as well as loss of data ratio as of the mobility of nodes [11]. This proposed model provided help in adjusting the topology update interval which resultant into reduction in data loss. In order to identify the tolerance of a node, Buffer zone was also introduced when mobile by transmitting a node in its range. The proposed scheme was extended as well as considered in view of indentifying the buffer zone effect. The model proposed in this paper provided a better scheme particularly for mobile node. The Cluster head nodes election process was not included in mobility of a node that reduced overall performance of the network.

Energy Efficient LEACH protocol with Reduced Data Redundancy in Wireless Sensor Network has been proposed in [5] by Dias D. This paper concentrates on redundancy of data. Sensors in network forwarded the sensed information to the cluster head in a particular time interval. The proposed technique concluded that the redundancy of data has been reduced by verifying the data in $\mathrm{CH}$ aggregation. Consequently, it reduced the consumption of energy of Cluster head. Moreover, it has been concluded that $\mathrm{CH}$ requires high energy in view of verifying the redundancy of data. Selection of High energy $\mathrm{CH}$ can increase the lifetime of the network. This scheme consumed high energy for large scale networks as the transmission of data rate was high and cluster head was busy in checking the redundancy rather than acquiring the data.

Liu et al. have worked on load balancing and energy minimization and compared their Energy-Aware Routing Protocol (EAP) named scheme with existing LEACH protocol [3]. In the process of $\mathrm{CH}$ election a number of parameters has been used by EAP scheme. In comparison to LEACH, an energy consumption is handled in a better way by Heterogeneous. The problem of area coverage is rectified after being identified by introduction of Intra-cluster coverage. There in increase in node capability using EAP that helps in acknowledged and testing node density. To reach at BS using LEACH there is need of higher distance to be covered or need of less energy to elect it on the other end using of $\mathrm{CH}$ election EAP proposed scheme able to achieve better results. There is 
quick reduction in energy of $\mathrm{CH}$ as in EAP cluster formation is not distributed that results in network lifetime reduction.

$\mathrm{Xu}$ et al. have discussed HEED protocol extension name it balanced energy-efficiency (BEE) clustering algorithm [4]. The cluster heads local density is considered to provide network coverage using BEE. There is single hop communication that's why in case of long range transmission there is more consumption of energy. The clustering algorithm name as balanced energy-efficiency Multihop (BEEM) which is the BEE multihop version is also discussed in this paper which gives improved performance as compared to existing BEE. The cluster heads optimal set are found by clustering using particle swarm optimization protocol is discussed by Elhabyan et al. that results in energy efficiency and network coverage maximization [5]. Ye et al. have used energy-efficient clustering scheme (EECS) that uses a competitive algorithm cluster head without exchange of iterations of message [6]. The increase residual energy is used to choose cluster head for which a HEED and LEACH is extended in it. The IBLEACH and HEED are most popular protocol among all above discussed protocols as balanced energy consumption is provided using it over existing nodes in each cluster that results in increase network lifetime. The network lifetime get further increased with the help of distributing workload efficiently. The different experiments are performed for comparing the results of proposed method with IBLEACH and HEED protocol.The LEACH protocol extension hybrid energy efficient distributed (HEED) clustering protocol is discussed in paper [7] that work based on nodes degree and residual energy for cluster heads selection. In terms of iterations a clustering process is carried out in this protocol. An iteration ends at cluster heads to BS after being started the data transmission from cluster selection. The probability of nodes that has not been used as cluster heads is more to become a cluster head in an iteration process. In terms of exchanged messages and processing cycles a number of overheads are low but on another end for cluster rebuilding there is need of extra energy. The intrabalanced LEACH (IBLEACH) protocol is processed for improving LEACH by Salim et al. [8]. In data collection as well as selection of cluster head the energy consumption gets balanced inside the clusters.

The LEACH based threshold-based cluster head replacement scheme name as T-LEACH protocol is discussed by Hong et al. that helps in to be selected cluster heads minimization [9]. The residual energy threshold is used for cluster heads minimization and an adaptive decentralized re-clustering protocol (ADRP) is discussed by Bajaber et al. in [10]. In this a nodes residual energy is used along with cluster head selection average energy. The threshold based protocol name ECLEACH cluster based protocol is discussed by Bsoul et al. [11]. The residual energy of sensors to be considered for cluster heads, their distance from other sensors, and residual energy of other sensors three parameters are used for cluster heads selection. In order to have better distribution there is minimum distance among cluster heads and energy-efficient multi-level clustering (EEMC) approach is discussed in Jin et al. [12]. The cluster heads determination is done on the next level to their members and itself and before that energy information and location is collected using BS. The heads is determined after collecting the information of each head and until a last tree level is not reached this process will be repeated.

\section{DIFFERENCE BETWEEN CLUSTERING ALGORITHM}

There is a variety of clustering algorithm that is applicable to wireless sensor networks to achieve energy efficient clustering. The difference between available clustering algorithms is shown in Table 1.

The clustering techniques are compared on the basis of the cluster head selection criteria and cluster formation for non $\mathrm{CH}$ nodes. Considering, review of different clustering algorithms proposed by several researchers have been discussed which concludes their outcomes in different parameters shown in table below.

Table 1.Review of different clustering algorithms [12]

\begin{tabular}{|c|c|c|c|c|c|c|c|c|c|c|}
\hline & \multicolumn{6}{|c|}{ Cluster head Selection } & \multicolumn{4}{|c|}{ Cluster Formation for Non-CH nodes } \\
\hline Scheme & Networking & $\begin{array}{l}\text { Distance } \\
\text { from } \\
\text { CH to } \\
\text { the BS }\end{array}$ & $\begin{array}{l}\text { CH } \\
\text { uniform } \\
\text { Distribution }\end{array}$ & $\begin{array}{l}\text { Over- } \\
\text { head } \\
\text { in CH } \\
\text { selection }\end{array}$ & $\begin{array}{l}\text { Residual } \\
\text { Energy }\end{array}$ & Delay & $\begin{array}{l}\text { Distance } \\
\text { non-CH } \\
\text { to } \mathrm{CH}\end{array}$ & $\begin{array}{l}\text { Cluster } \\
\text { Size }\end{array}$ & Hops & $\begin{array}{l}\text { Over- } \\
\text { head to } \\
\text { join a } \\
\text { cluster }\end{array}$ \\
\hline LEACH & Distributed & No & No & Low & No & Low & Yes & No & 1 & Low \\
\hline $\begin{array}{l}\text { TL- } \\
\text { LEACH }\end{array}$ & Distributed & No & No & Medium & No & Medium & Yes & No & 2 & High \\
\hline EECS & Distributed & Yes & Yes & Low & Yes & Low & Yes & Yes & 1 & Low \\
\hline EEUC & Distributed & Yes & Yes & Low & Yes & Low & Yes & Yes & $\mathrm{K}$ & Low \\
\hline
\end{tabular}




\begin{tabular}{|l|l|l|l|l|l|l|l|l|l|l|}
\hline MRPVC & Distributed & Yes & Yes & Low & Yes & Low & Yes & No & 1 & Low \\
\hline PEACH & Distributed & No & No & Low & No & Medium & Yes & No & K & Low \\
\hline
\end{tabular}

\section{CONCLUSION AND FUTURE SCOPE}

WSN performs the data transmission on the basis of the routing and clustering. For the purpose of clustering the Cluster Heads $(\mathrm{CH})$ are elected. The $\mathrm{CH}$ is responsible for the data delivery to the base station. There are lots of issues that came across during the data transmission and load balancing is one of those issues. This study highlights the various clustering algorithms that are used for solving different issues. From the survey that has been conducted in this work, it has been concluded that clustering is most suitable and reliable technique for large scale wireless sensor networks as it reduces communication overhead as well as exploits data aggregation. There are number of clustering algorithms have been proposed by several researchers to enhance the energy efficiency of the sensor nodes. However, the consumption of energy while cluster formation and maintenance is still high. The primary focus of the clustering algorithms is to maximize the network lifetime while considering several challenges like computation of optimal cluster size, determining the optimal frequency for cluster head rotation etc.

In future, a novel approach can be derived which can focus on balancing the load of the clusters while having a keen eye on consumption of energy in sensor nodes.

\section{REFERENCES}

[1] Bajaber, F., Awan, I., "Adaptive decentralized reclustering protocol for wireless sensor networks" Journal of Computer and System Sciences, Vol. 77, No. 2, Pp.282-292, 2011

[2] Bsoul, M., Al-Khasawneh, A., Abdallah, A. E., Abdallah, E. E., Obeidat, I.,"An energy-efficient threshold-based clustering protocol for wireless sensor networks" Wireless Pers Commun, Vol. 70, No. 1, Pp.99-112, 2013

[3] Bencan Gong, Layuan Li, Shaorong Wang, Xuejun Zhou, Multihop Routing Protocol with unequal Clustering for Wireless Sensor Networks International Colloquium on Computing, Communication, Control, and Management,(ISECS2008),Pp. 552-556, 2008

[4] C. Li, M. Ye, G. Chen, J. Wu, "An energy efficient unequal clustering mechanism for wireless sensor networks," in: Proceedings of 2005 IEEE International Conference on Mobile Adhoc and Sensor Systems Conference(MASS05), Washington, D.C., Pp. 604-611, November 2005.

[5] Dias, D., Jeberson, W., and Jeberson, K.,"Energy Efficient LEACH Protocol with reduced Data Redundancy in WSN", Estimation of Benefit-Cost Ratio under Composite Fish Farming in Village Ponds (Earthen) of Allahabad District,Vol. 71, No. 2, Pp. 1-4, 2016.

[6] Elhabyan, R.S. and Yagoub, M.C.E, "Particle swarm optimization protocol for clustering in wireless sensor networks: A realistic approach," Proc. 15th IEEE Int.
Conf. on Information Reuse and Integration (IRI), Redwood City, CA, Pp.345-350, 2014.

[7] Hong, J., Kook, J., Lee, S., Kwon, D., Yi, S., “T-LEACH: The method of threshold-based $\mathrm{CH}$ replacement for wireless sensor networks" Information Systems Frontiers, Vol. 11, No. 5, Pp.513-521, 2009.

[8] Jin, Y., Wang, L., Kim, Y., Yang, X., "EEMC: an energyefficient multi-level clustering algorithm for large-scale wireless sensor networks", Computer Networks, Vol. 52, Pp.542-562, 2008.

[9] Liu, M., Cao, J., Chen, G., and Wang, X.,“An energyaware routing protocol in wireless sensor networks", Sensors, Vol. 9, No. 1, Pp. 445-462, 2009.

[10] M. Ye, C. Li, G. Chen and J. Wu, “An Energy Efficient Clustering Scheme in Wireless Sensor Networks," Ad Hoc \& Sensor Wireless Networks,Vol.1, Pp.1-21,2006.

[11] Nayebi, A., and Sarbazi-Azad, H., "Performance modeling of the LEACH protocol for mobile wireless sensor networks", Journal of parallel and distributed computing, Vol. 71, No. 6, Pp. 812-821, 2011.

[12] OlutayoBoyinbode, Hanh Le, Audrey Mbogho, Makoto Takizawa and Ravi Poliah, "A Survey on Clustering Algorithms for Wireless Sensor Networks", $201013^{\text {th }}$ International Conference On Network Based Information Systems, Pp. 358-364, November 2010.

[13] S.Yi, J. Heo, Y. Cho, and J. Hong, "PEACH: powerefficient and adaptive clustering hierarchy protocol for wireless sensor networks", Computer Communications, Vol. 30, Pp. 2842-2852, October 2007.

[14] Salim, A., Osamy and W., Khedr, A. M.,"IBLEACH: intra-balanced LEACH protocol for wireless sensor networks", Wireless Networks, Vol. 20, Pp.1515-1525, 2014

[15] V. Loscri, G. Morabito and S. Marano, "A Two-Level Hierarchy for Low-Energy Adaptive Clustering Hierarchy", Proceedings of Vehicular Technology Conference 2005, Vol.3, Pp. 1809-1813, 2005

[16] W. Heinzelman, A. Chandrakasan and H.Balakrishnan, "Energy- Efficient Communication Protocol for Wireless Micro sensor Networks", Proceedings of the 33rd Hawaii International Conference on System Sciences (HICSS '00), Vol. 2, No. 10, January 2000.

[17] Xu, L., O'Hare, G.M.P., Collier, R., “A Balanced Energy-Efficient Multihop clustering scheme for Wireless Sensor Networks," Proc. 7th IFIP on Wireless and Mobile Networking Conf. (WMNC),Vilamoura, Pp.1-8, 2014.

[18] Ye, M., Li, C., Chen G., Wu, J., "EECS: an energy efficient clustering scheme in wireless sensor networks,' 
International Journal of Computer Applications (0975 - 8887)

Volume 180 - No.33, April 2018

Proc. 24th Int. Conf. on Performance, Computing, and Communications, Pp.535 -540, 2005.

[19] Younis, O., Fahmy, S.,"HEED: A Hybrid, EnergyEfficient, Distributed Clustering Approach for Ad Hoc
Sensor Networks", IEEE Transactions on Mobile computing,vol.3,No.4.Pp.366-379,2004 